\title{
Two-Step Incision for Periarterial Sympathectomy of the Hand
}

\author{
Seung Bae Jeon ${ }^{1}$, Hee Chang Ahn ${ }^{1}$, Yong Su Ahn², Matthew Seung Suk Choi ${ }^{3}$ \\ ${ }^{1}$ Department of Plastic and Reconstructive Surgery, Hanyang University Seoul Hospital, Hanyang University College of Medicine, Seoul; \\ ${ }^{2}$ START Plastic Surgery Clinic, Seoul; ${ }^{3}$ Department of Plastic and Reconstructive Surgery, Hanyang University Guri Hospital, Hanyang \\ University College of Medicine, Guri, Korea
}

Background Surgical scars on the palmar surface of the hand may lead to functional and also aesthetic and psychological consequences. The objective of this study was to introduce a new incision technique for periarterial sympathectomy of the hand and to compare the results of the new two-step incision technique with those of a Koman incision by using an objective questionnaire.

Methods A total of 40 patients ( 17 men and 23 women) with intractable Raynaud's disease or syndrome underwent surgery in our hospital, conducted by a single surgeon, between January 2008 and January 2013. Patients who had undergone extended sympathectomy or vessel graft were excluded. Clinical evaluation of postoperative scars was performed in both groups one year after surgery using the patient and observer scar assessment scale (POSAS) and the Wake Forest University rating scale.

Results The total patient score was 8.59 (range, 6-15) in the two-step incision group and 9.62 (range, 7-18) in the Koman incision group. A significant difference was found between the groups in the total PS score $(P$-value $=0.034)$ but not in the total observer score. Our analysis found no significant difference in preoperative and postoperative Wake Forest University rating scale scores between the two-step and Koman incision groups. The time required for recovery prior to returning to work after surgery was shorter in the two-step incision group, with a mean of 29.48 days in the two-step incision group and 34.15 days in the Koman incision group $(\mathrm{P}=0.03)$.

Conclusions Compared to the Koman incision, the new two-step incision technique provides better aesthetic results, similar symptom improvement, and a reduction in the recovery time required before returning to work. Furthermore, this incision allows the surgeon to access a wide surgical field and a sufficient exposure of anatomical structures.

Keywords Raynaud disease / Sympathectomy
Correspondence: Hee Chang Ahn Department of Plastic and

Reconstructive Surgery, Hanyang University Medical Center, 345 Wangsimni-gil, Sungdong-gu, Seoul 04763, Korea

Tel: $+82-2-2290-8560$

Fax: +82-2-2295-7671

E-mail: ahnhc@hanyang.ac.kr

We are grateful to Jee Won Shin, medical illustrator, for the illustrations.

This article was presented at the Research \& Reconstructive Forum on April 3-4, 2014 in Busan, Korea.

No potential conflict of interest relevant to this article was reported.

Received: 13 May 2015 • Revised: 28 Aug 2015 • Accepted: 24 Sep 2015

pISSN: 2234-6163 • elSSN: 2234-6171 • http://dx.doi.org/10.5999/aps.2015.42.6.761 • Arch Plast Surg 2015;42:761-768

\section{INTRODUCTION}

The treatment of Raynaud's symptoms of cold intolerance, pain, and ulceration that are refractory to oral medication is an extremely difficult clinical problem [1]. Historically, cervicothoracic sympathectomy was a common treatment if conservative

Copyright (C) 2015 The Korean Society of Plastic and Reconstructive Surgeons

This is an Open Access article distributed under the terms of the Creative Commons Attribution Non-Commercial License (http://creativecommons.org/

licenses/by-nc/3.0/) which permits unrestricted non-commercial use, distribution, and reproduction in any medium, provided the original work is properly cited.

www.e-aps.org 
treatment of symptoms was unsuccessful [2]. However, this treatment provided only temporary improvement, and in 1980 Flatt et al. suggested the resection of the periarterial sympathetic fibers and digital nerve branches to the common and proper digital arteries. This technique has provided significant improvement in selected patients $[3,4]$. However, the associated incisions on the palmar surface of the hand typically lead to redness, pain, edema, tenderness, and aesthetic problems. Surgical scars on the palm may lead to not only functional but also aesthetic and psychological consequences, particularly if they are located in areas of the body that are actively moving and are exposed instead of covered by clothing $[5,6]$. Therefore, it is necessary to optimize scar formation by using appropriate incision techniques and performing a careful follow-up to check the healing process. Minimal incisions made for aesthetic purposes may lead to insufficient exposure of the operative field. Therefore, we have performed a new incision technique over several recent years in an attempt to obtain a sufficient operative field and improved aesthetic outcomes. The objective of this study was to introduce a new incision technique for periarterial sympathectomy of the hand and to compare the results of the new two-step incision technique with those of the Koman incision by using objective questionnaire. We hypothesized that periarterial sympathectomy of the palm performed with a two-step incision would result in a more aesthetically acceptable and functional scar, reduced postoperative pain, and earlier mobilization and discharge from the hospital.

\section{METHODS}

A total of 40 patients (17 men and 23 women) with intractable Raynaud's disease or syndrome underwent surgery in our hospital, conducted by a single surgeon, between January 2008 and January 2013. A retrospective study of periarterial sympathectomy, based on chart review, was designed and conducted. All patients had a diagnosis of Raynaud's disease or syndrome by a rheumatologist. They suffered from pain, ulcers, or gangrene in the affected digits and were unresponsive to pharmacologic or other conservative therapies. Pharmacologic interventions include alpha-blocking agents, beta-blocking agents, and calcium channel-blocking agents. We evaluated preoperative arteriography to assist with the decision for arterial reconstruction, and if possible, revascularization procedures were also performed. In all cases in our study, angiography showed multifocal arterial pathologic changes. A consecutive series of 40 Raynaud's patients managed with periarterial sympathectomy was comprised of patients receiving either a two-step incision or a Koman incision on the palm, as well as ulnar and radial incisions on the wrist. Patients who had undergone extended sympathectomy or vessel graft were excluded. The two-step incision technique was used in 28 selected patients between January 2008 and January 2013. The twelve patients who had a Koman incision served as the control group for comparison. All patients were admitted the day before surgery, which is the present practice at our institution. We analyzed the period that elapsed before return to work in order to evaluate the functional outcome.

\section{Incision methods}

In the Koman incision group, the incision was made in the middle of the palm as a single transverse line (Fig. 1A). In the twostep incision group, the incision consisted of two separate incisions in the proximal and distal palmar creases. The distal incision was made on the distal palmar crease from the proximal continuation of the center of the long finger to that of the little finger. The proximal incision was made on the proximal palmar crease from the proximal continuation of the radial side of the index finger to that of the ring finger. These incision lines can be modified to expose specific structures such as narrowed arteries (Fig. 1B). In all cases, longitudinal incisions were also made at the volar wrist over the radial and ulnar arteries. A straight skin incision over the ulnar artery, aligned with the crest of the ulna, was made from the proximal wrist crease to $5 \mathrm{~cm}$ more proximally (Fig. 1). Next, the fascia overlying the arteries was incised and flexor carpi ulnaris was retracted laterally and flexor digitorum superficialis was retracted medially. Another skin incision for the radial artery, aligned with the edge of the brachioradialis muscle, was extended from the proximal wrist crease to $5 \mathrm{~cm}$

\section{Fig. 1. Incision method of periarterial sympathectomy}

(A) Incision method for periarterial sympathectomy in the hand. Koman incision (red), ulnar and radial incision (blue). (B) Incision method for periarterial sympathectomy in the hand. Two-step incision (red), ulnar and radial incision (blue).
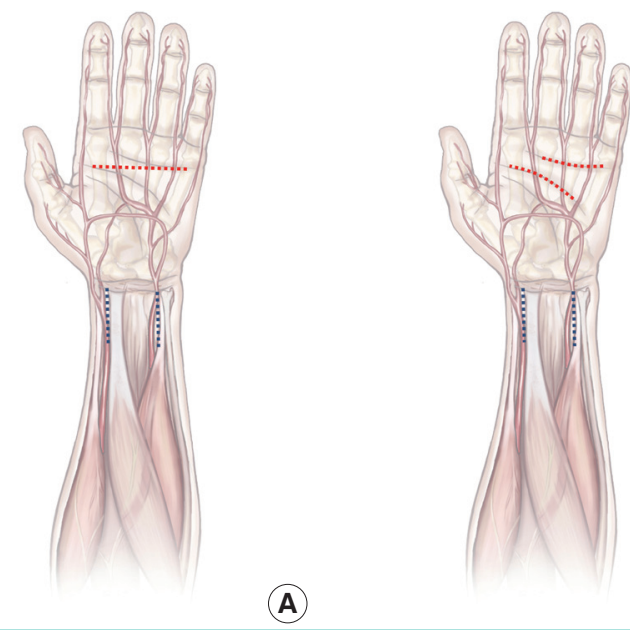
more proximally. Next, the fascia overlying the arteries was incised and brachioradialis was retracted laterally and flexor carpi radialis was retracted medially.

\section{Postoperative management}

Dressings were applied to minimize scar formation. Steri-strips were applied postoperatively along the sutured incision line after the application of antibiotic powder. Each strip was overlapped and attached without ointment so it could reinforce the subcuticular and simple interrupted sutures. After the stitches were removed, placement of strips was continued for six months.

\section{Patient and observer scar assessment scale}

Clinical evaluation of postoperative scars was performed in both groups one year after surgery and postoperative management with self-drying silicone gel and adhesive strips using the patient and observer scar assessment scale (POSAS). The POSAS consists of two subscales: the patient scale (PS) and the observer scale (OS). The PS was compiled by the patient, who rated scar pain, itch, color, stiffness, thickness, and surface. All items were evaluated on a numerical rating scale ranging from 1 to 10 (with 10 indicating the worst imaginable scar or sensation). The observer completed the OS using the same scale and rated scar vascularity, pigmentation, thickness, relief, pliability, and surface area (Table 1). The OS was evaluated based on clinical experience and on references in the literature such as the Vancouver scale. The total PS and OS scores were obtained by adding the scores of each of the six items (range, 6-60), with higher scores indicating the worst imaginable scar. Both the observer and the patient finally gave their overall opinion on the appearance of the scar, assigning a score ranging from 1 to $10[7,8]$.

\section{Wake Forest University rating scale}

Symptoms such as pain, numbness, and cold intolerance were assessed in each patient before and after undergoing periarterial sympathectomy using the Wake Forest University rating scale ( 0 , none; 1 , mild; 2 , moderate; 3 , severe), with a higher score indicating a greater intensity of symptoms.

\section{Statistical analysis}

Statistical analysis was performed using PASW ver. 18.0 (SPSS Inc., Chicago, IL, USA). The Mann-Whitney U test was used to compare the POSAS and Wake Forest University rating scale scores between the two groups. The Wilcoxon signed-rank test was used for comparing preoperative and postoperative Wake Forest University rating scale scores in each group. An independent $t$-test was used to compare the periods of hospitalization and recovery before return to work between the two groups. A P-value of less than 0.05 was considered statistically significant.

\section{RESULTS}

Our patients were aged between 27 and 74 years, with a mean age of 53.1 years. The majority of them were in their sixth and seventh decades (33\% and 30\%, respectively). $42 \%$ of patients were male. The number of smokers was five (three in the twostep incision group and two in the Koman incision group).

\section{Table 1. The patient and observer scar assessment scale}

\begin{tabular}{|c|c|c|c|c|}
\hline \multicolumn{5}{|l|}{ A. Patient scale } \\
\hline & & Normal skin & 1234567890 & Worst scar imaginable \\
\hline & Has the scar been painful the past few weeks? & & 0000000000 & \\
\hline & Has the scar been itching the past few weeks? & & 0000000000 & \\
\hline & Is the scar color different from the color of your normal skin at present? & & 0000000000 & \\
\hline & Is the stiffness of the scar different from your normal skin at present? & & 0000000000 & \\
\hline & Is the thickness of the scar different from your normal skin at present? & & 0000000000 & \\
\hline & Is the scar more irregular than your normal skin at present? & & 0000000000 & \\
\hline & & Normal skin & 1234567890 & Worst scar imaginable \\
\hline Overall opinion & & & 0000000000 & \\
\hline \multicolumn{5}{|l|}{ B. Observer scale } \\
\hline & & Normal skin & 1234567890 & Worst scar imaginable \\
\hline & Vascularity & & 0000000000 & \\
\hline & Pigmentation & & 0000000000 & \\
\hline & Thickness & & 0000000000 & \\
\hline & Relief & & 0000000000 & \\
\hline & Pliability & & 0000000000 & \\
\hline & Surface area & & 0000000000 & \\
\hline & & Normal skin & 1234567890 & Worst scar imaginable \\
\hline Overall opinion & & & 0000000000 & \\
\hline
\end{tabular}




\section{Patient and observer scar assessment scale}

The POSAS scores were lower on almost all of the items in the two-step incision group than in the Koman incision group. Of note, the total PS score was 8.59 (range, 6-15) in the two-step incision group, whereas it was 9.62 (range, 7-18) in the Koman incision group. The total OS score was 11.79 (range, 7-20) in the two-step incision group and 11.84 (range, 8-20) in the Koman incision group. A significant difference was found between the groups in total PS score $(\mathrm{P}=0.034)$, but not in total OS score $(\mathrm{P}=0.419)$ (Table 2). There were significant differences in PS scores on the parameters of pain $(\mathrm{P}=0.028)$, color $(\mathrm{P}=$ 0.045), and overall opinion ( $\mathrm{P}=0.001)$ (Table 3$)$ and in $\mathrm{OS}$ score in overall opinion $(\mathrm{P}=0.003)$ (Table 4$)$. The other parameters were not significantly different between groups.

\section{Wake Forest University rating scale}

In the Koman incision group, evaluation of patient symptoms with the Wake Forest University rating scale indicated that pa-

\section{Table 2. Total POSAS scores in the Koman and two-step} incision groups

\begin{tabular}{|lccc|}
\hline Scale & Koman incision & Two-step incision & P-value \\
\hline Patient scale & $9.62(7-18)$ & $8.59(6-15)$ & 0.034 \\
Observer scale & $11.84(8-20)$ & $11.79(7-20)$ & 0.419 \\
\hline \multicolumn{4}{l}{ Values are presented as the mean (range). } \\
\hline \multicolumn{4}{l}{ POSAS, patient and observer scar assessment scale. } \\
\hline
\end{tabular}

\section{Table 3. Total patient scale scores}

\begin{tabular}{|lccc|}
\hline Variable & Koman incision & Two-step incision & P-value \\
\hline Pain & $2.34(1-5)$ & $1.31(1-3)$ & 0.028 \\
Itch & $1.29(1-4)$ & $1.25(1-3)$ & 0.449 \\
Color & $2.78(1-7)$ & $1.9(1-4)$ & 0.045 \\
Stiffness & $1.56(1-5)$ & $1.49(1-5)$ & 0.966 \\
Thickness & $1.34(1-5)$ & $1.35(1-5)$ & 0.282 \\
Surface & $1.31(1-4)$ & $1.29(1-4)$ & 0.802 \\
Overall opinion & $3.08(1-7)$ & $2.11(1-4)$ & 0.001 \\
\hline \multicolumn{4}{l}{ Values are presented as the mean (range). } \\
\hline
\end{tabular}

\section{Table 4. Total observer scale scores}

\begin{tabular}{|lccc|}
\hline Variable & Koman incision & Two-step incision & P-value \\
\hline Vascularity & $1.34(1-4)$ & $1.44(1-4)$ & 0.112 \\
Pigmentation & $4.23(3-7)$ & $4.14(2-6)$ & 0.242 \\
Thickness & $1.27(1-5)$ & $1.26(1-5)$ & 0.977 \\
Relief & $2.23(1-6)$ & $2.15(1-6)$ & 0.454 \\
Pliability & $1.77(1-4)$ & $1.80(1-5)$ & 0.784 \\
Surface area & $1(1-1)$ & $1(1-1)$ & 0.465 \\
Overall opinion & $2.14(2-5)$ & $1.93(1-4)$ & 0.003 \\
\hline \multicolumn{4}{l}{ Values are presented as the mean (range). } \\
\hline
\end{tabular}

tients experienced reduced pain $(\mathrm{P}<0.001)$ and numbness $(\mathrm{P}=0.038)$ after periarterial sympathectomy surgery. Cold intolerance, however, was not reduced significantly after the operation. In the two-step incision group, patients experienced reduced pain $(\mathrm{P}<0.001)$ and numbness $(\mathrm{P}=0.028)$ after the surgery, but cold intolerance was not significantly reduced.

The preoperative scores under the Wake Forest University rating scale showed that the patients reported pain at a mean level of 2.41 in the Koman incision group and 2.27 in the two-step incision group. The mean numbness score was 1.75 in the Koman incision group and 1.82 in the two-step incision group, and the mean cold intolerance score was 2.23 in the Koman incision and 2.43 in the two-step incision group. Our analysis found no significant difference between the preoperative scores in the Koman and two-step incision groups.

One year after sympathectomy, the Wake Forest University rating scale scores demonstrated that the patients reported pain at a mean level of 0.67 in the Koman incision group and 0.56 in the two-step incision group. The mean numbness score was 0.89 in the Koman incision group and 0.71 in the two-step incision group, and the mean cold intolerance score was 1.54 in the Koman incision group and 1.67 in the two-step incision group. There was no significant difference in postoperative scores between the Koman and two-step incision groups (Fig. 2).

\section{Period of hospitalization and return to work}

The average postoperative hospitalization period was 13.74 days in the Koman incision group and 11.42 days in the two-

\section{Fig. 2. Wake Forest University rating scale}

The Wake Forest University rating scale before and after the operation in both groups. Koman incision group (blue bars) and two-step incision group (orange bars). Pre-OP., preoperative; Post-OP., postoperative.

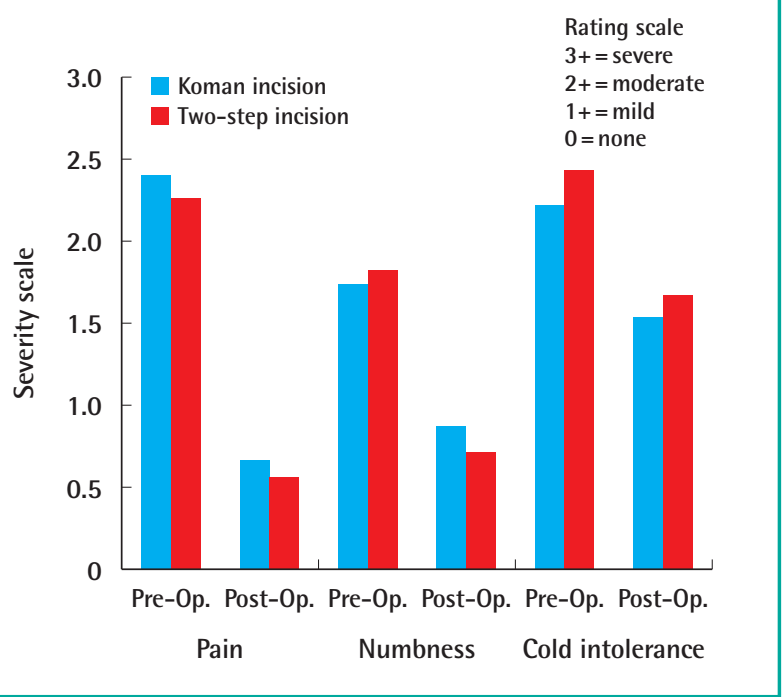


step incision group, and these periods were not significantly different. The period of recovery prior to returning to work was different for the two groups, with a mean of 34.15 days in the Koman incision group and 29.48 days in the two-step incision group $(\mathrm{P}=0.03)$ (Table 5).

\section{Case 1}

A 64-year-old woman had been suffering from skin necrosis on her right second finger for several months. The patient had a history of Raynaud's syndrome, scleroderma, dermatomyositis and pulmonary fibrosis. Angiography showed multifocal stenosis over all proper digital arteries in the right hand. Conservative treatment was attempted without success. Periarterial sympathectomy was performed over the common digital arteries using the Koman incision and over the ulnar and radial arteries. Digital artery reconstruction with the superficial vein in the wrist was performed over the ulnar side of the right second finger. Cyanosis improved and the pain decreased post-surgery. Capillary refilling also improved. The patient was discharged on the

Table 5. Mean duration for recovery before return to work in Koman incision and two-step incision groups

\begin{tabular}{|lccc|}
\hline Variable & $\begin{array}{c}\text { Koman } \\
\text { incision }\end{array}$ & $\begin{array}{c}\text { Two-step } \\
\text { incision }\end{array}$ & P-value \\
\hline Period of hospitalization & $13.74(2.34)$ & $11.42(1.89)$ & 0.068 \\
$\begin{array}{l}\text { Period of recovery prior to } \\
\text { return to work }\end{array}$ & $34.15(5.64)$ & $29.48(4.68)$ & 0.030 \\
\hline \multicolumn{3}{l}{ Values are presented as the mean (standard deviation). } \\
\hline
\end{tabular}

13th postoperative day. The patient was treated according to the above-mentioned wound and scar care protocol for six months. The wound healed well but a clearly evident transverse scar was formed in the palm (Fig. 3).

\section{Case 2}

A 36-year-old woman had been suffering from skin necrosis of the second and third digits for several years. The patient also suffered from sensitivity to cyanosis in cold temperatures. Periarterial sympathectomy was performed over the common digital arteries using the two-step incision and ulnar and radial incisions. Postoperatively, her symptoms were improved. The patient was discharged on the 11th postoperative day and treated according to the wound care protocol for six months. After one year, the scar was almost invisible and folded with the existing palmar crease (Fig. 4).

\section{DISCUSSION}

Digital ischemia with subsequent gangrene and tissue necrosis caused by Raynaud's syndrome remains a difficult treatment problem for rheumatologists and hand surgeons $[9,10]$. Although several pharmacologic agents have been used successfully to improve symptoms, some patients remain refractory to medical management $[11,12]$. During the past several decades, periarterial sympathectomy has been used as a treatment option for improving the quality of life for patients suffering from severe Raynaud syndrome $[13,14]$. Postoperative pain and scars

Fig. 3. Koman incision method with ulnar and radial incision

(A) Preoperative design by the Koman incision method on the palm and ulnar and radial incision on the wrist. (B) Postoperative results after three days. (C) Postoperative results after one year.
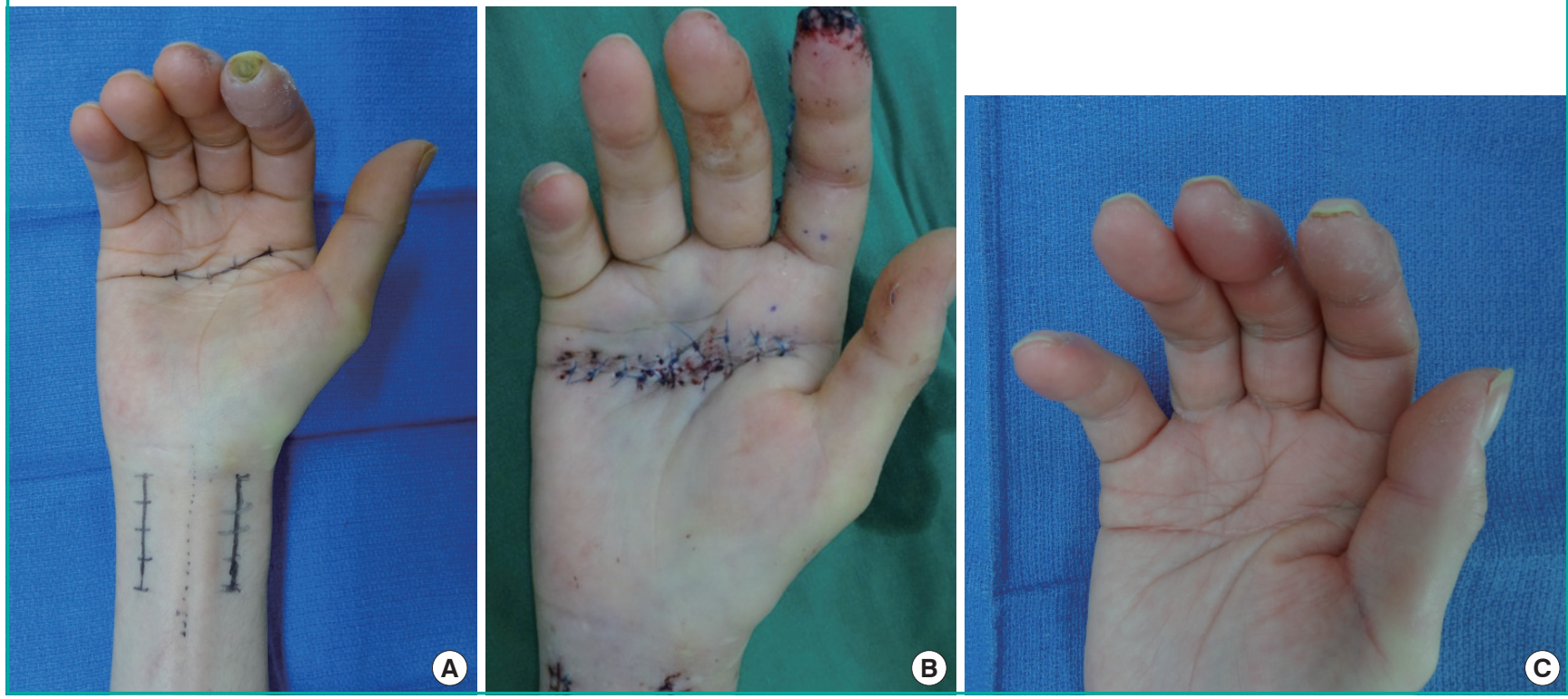
Fig. 4. Two-step incision method with ulnar and radial incision

(A) Preoperative design by the two-step incision method on the palm and ulnar and radial incision on the wrist. (B) Postoperative results after three days. (C) Postoperative results after one year.
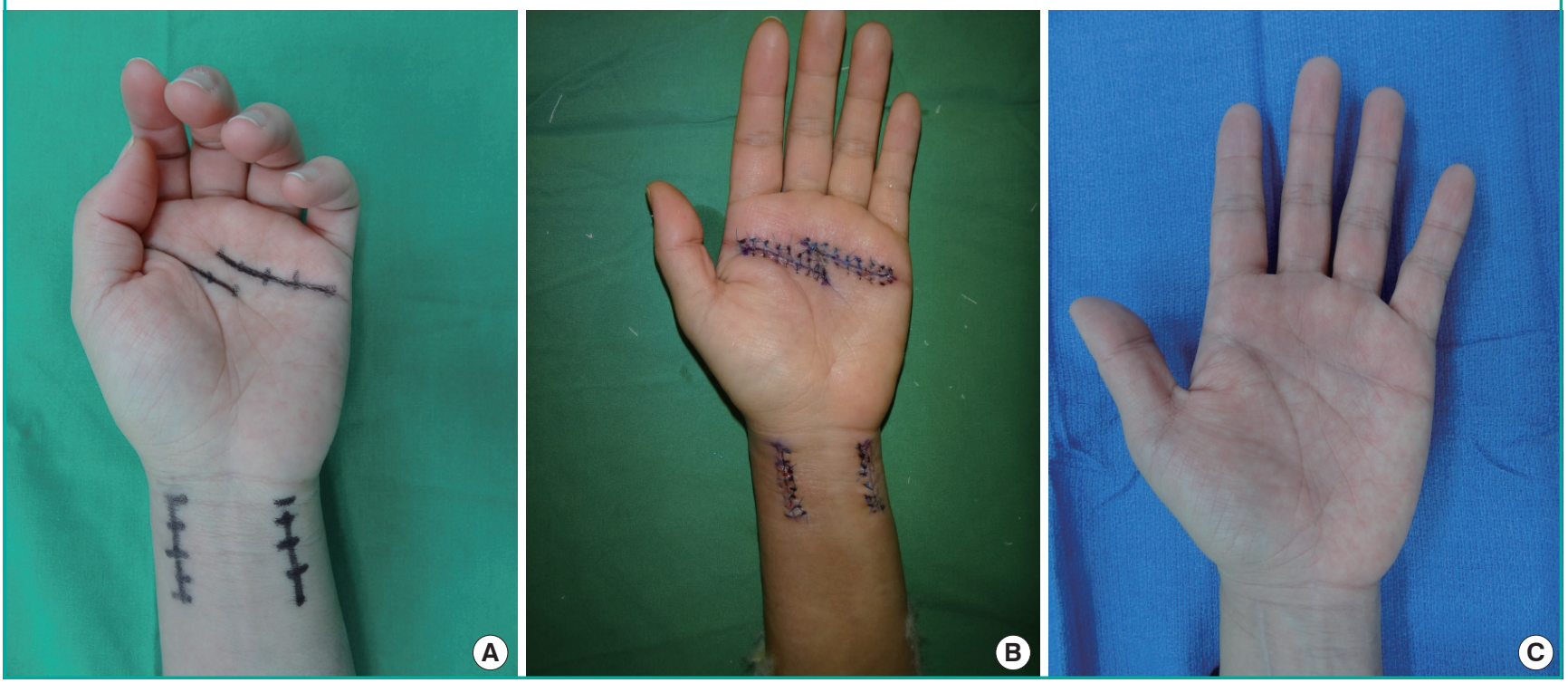

are known complications of the Koman incision for periarterial sympathectomy in the palm. These incisions leave a scar in the middle of the palm, an area subject to crease formation when making a fist. In some cases, an extra incision is required for visualizing the proximal common digital artery or proper digital artery. To the best of our knowledge, this retrospective study is the first to test whether periarterial sympathectomy in the palm performed through a two-step incision has any benefits over the Koman incision technique.

To reduce confounding variables, a single surgeon performed all aspects of the procedure, using the same operative techniques and the same protocol for wound dressing and scar management in all patients. This is important, because methods of dressing and scar management significantly affect scar formation [7].

Compared with the Koman incision method, our method of incision on the palmar crease offers the following advantages. First, the two-step incision conceals the scar by being placed at the proximal and distal palmar crease. Traditional incision inevitably creates a scar that is visually distinct from the palmar crease. Second, our method involves two separate incisions, which allows more extensive exposure of anatomical structures than the Koman incision, especially of the superficial palmar arch and common digital arteries. Third, the two separated curved incisions disperse the vector of the scar in all directions, which can reduce its impact on the range of motion of the finger. Thus, as well as palmar spreading, the post-surgical outcome results in increased function and less pain when patients make a fist and unfold the palm. As there is therefore very little effect of hand movement on wound healing, the stitches can be removed early and patients can mobilize and return to work more quickly.

In this study, wrist incision for ulnar and radial artery commenced from, and skin was preserved distal to, the proximal wrist crease. This was intended to reduce scar contracture and web formation in the wrist, and it did not interfere with the operative field.

Although reduced pain is one of the benefits that have been cited for this surgical approach, none of the previously reported studies have directly measured pain as an outcome. For this reason, our two-step incision was objectively assessed using several parameters, including pain. The patient and observer scar assessment scale is reported in the literature as a verified tool for the evaluation of linear scars $[15,16]$. The incision method for the wrist does not differ between the two-step and Koman incision methods.

The damaging effect of cigarette smoking on digital perfusion and wound healing has been reported in several studies $[17,18]$. Despite active counseling, five of 40 patients continued to smoke during the follow-up period in the present study (three in the two-step incision group and two in the Koman incision group). Three of these five patients had a healing period for a digital ulcer of more than six months' duration (two in the twostep incision group and one in the Koman incision group). Two of these five patients had active ulcers at follow-up (one in each incision group). These results suggest a poor long-term out- 
come for patients who continue to smoke. We strongly encourage smoking cessation but will perform periarterial sympathectomy in patients who continue to smoke because we consider it to be a salvage procedure.

The incidence of a single transverse palmar crease is $7 \%-10 \%$ in the general population [19]. Because this would be a confounding factor in our analysis, patients with a single transverse palmar crease were not included in this study. In these cases, a Koman incision was performed on the palmar crease, which was not effectively different to the procedure for a two-step incision, which is also performed on the palmar crease, and so it had a similar effect on scar formation.

In the present study, patients in both groups reported decreased symptoms, most significantly in the area of pain and numbness. These changes were associated with significant improvement in digital micro-vascular perfusion, resulting in a resolution of ischemia. However, cold intolerance did not improve significantly in either group. The fact that cold intolerance, as assessed by the Wake Forest University rating scale, did not change after periarterial sympathectomy is not surprising when considering the unchanged total blood flow to the digits. Johnston et al. reported that cold intolerance persisted in spite of adequate sympathetic denervation [20]. This indicates that nutritional blood flow and cutaneous perfusion are not enough to improve cold intolerance following periarterial sympathectomy. An additional vessel graft could perhaps improve cold intolerance, although additional studies would be necessary to determine this.

The postoperative outcomes in the Koman incision group and the two-step incision group were similar. We found that most patients' wounds recovered well and symptoms subsided. Only two men (one in each incision group) reported no symptom reduction and continued to experience digital ulcers and substantial pain. None of the patients suffered from major complications. Taken together, these observations indicate that the twostep incision could replace the Koman incision method.

Using the new incision technique, the scar at the palm was nearly invisible, being well integrated into the natural palmar crease at one year after surgery. Both patients and surgeons were more satisfied with the cosmetic appearance of the palm after wound healing with the two-step incision method than with the Koman incision method. In our experience, this new approach provides not only a more natural appearance, but also greater functional improvement. Furthermore, it results in sufficient exposure of anatomical structures and provides a wide surgical field.

\section{REFERENCES}

1. Koman LA, Urbaniak JR. Ulnar artery insufficiency: a guide to treatment. J Hand Surg Am 1981;6:16-24.

2. Miller LM, Morgan RF. Vasospastic disorders: etiology recognition, and treatment. Hand Clin 1993;9:171-87.

3. Jones NF. Acute and chronic ischemia of the hand: pathophysiology, treatment, and prognosis. J Hand Surg Am 1991;16:1074-83.

4. Wilgis EF. Evaluation and treatment of chronic digital ischemia. Ann Surg 1981;193:693-8.

5. Bruner JM. Optimum skin incisions for the surgical relief of stenosing tenosynovitis in the hand. Plast Reconstr Surg 1966;38:197-201.

6. Bruner JM. The zig-zag volar-digital incision for flexor-tendon surgery. Plast Reconstr Surg 1967;40:571-4.

7. Bianchi FA, Roccia F, Fiorini P, et al. Use of Patient and Observer Scar Assessment Scale for evaluation of facial scars treated with self-drying silicone gel. J Craniofac Surg 2010; 21:719-23.

8. Draaijers LJ, Tempelman FR, Botman YA, et al. The patient and observer scar assessment scale: a reliable and feasible tool for scar evaluation. Plast Reconstr Surg 2004;113:19605.

9. Drake DB, Kesler RW, Morgan RF. Digital sympathectomy for refractory Raynaud's phenomenon in an adolescent. J Rheumatol 1992;19:1286-8.

10. Barral X, Favre JP, Gournier JP, et al. Late results of palmar arch bypass in the treatment of digital trophic disorders. Ann Vasc Surg 1992;6:418-24.

11. Ward WA, Van Moore A. Management of finger ulcers in scleroderma. J Hand Surg Am 1995;20:868-72.

12. Landry GJ, Edwards JM, Porter JM. Current management of Raynaud's syndrome. Adv Surg 1996;30:333-47.

13. Balogh B, Mayer W, Vesely M, et al. Adventitial stripping of the radial and ulnar arteries in Raynaud's disease. J Hand Surg Am 2002;27:1073-80.

14. Ortensi A, Salsano F, Trinchi S, et al. Microsurgical distal sympathectomy in chronic vasospastic syndromes of the hand. Int Surg 2005;90:88-92.

15. van de Kar AL, Corion LU, Smeulders MJ, et al. Reliable and feasible evaluation of linear scars by the Patient and $\mathrm{Ob}$ server Scar Assessment Scale. Plast Reconstr Surg 2005; 116: 514-22.

16. Durani P, McGrouther DA, Ferguson MW. Current scales for assessing human scarring: a review. J Plast Reconstr Aesthet Surg 2009;62:713-20.

17. Fulcher SM, Koman LA, Smith BP, et al. The effect of trans- 
dermal nicotine on digital perfusion in reformed habitual smokers. J Hand Surg Am 1998;23:792-9.

18. Morecraft R, Blair WF, Brown TD, et al. Acute effects of smoking on digital artery blood flow in humans. J Hand Surg Am 1994;19:1-7.
19. Smallpeice PADV. The single transverse palmar crease in infants and children. Dev Med Child Neurol 1963;5:491-6.

20. Johnston EN, Summerly R, Birnstingl M. Prognosis in raynaud's phenomenon after sympathectomy. Br Med J 1965;1: 962-4. 